\section{Non-puerperal mastitis masking pre-existing breast malignancy: importance of follow-up imaging}

\author{
Jin Kyung $A n^{1}$, Jeong Joo Woo', Seung A Lee ${ }^{2}$ \\ Departments of ${ }^{1}$ Radiology and ${ }^{2}$ Surgery, Eulji General Hospital, Eulji University School of \\ Medicine, Seoul, Korea
}

Mastitis is an inflammatory condition of the breast with common symptoms of pain, swelling, erythema, warmth, and fever. Diagnosis of mastitis is easily made on the basis of typical symptoms and ultrasonographic findings, such as diffusely increased echogenicity of the parenchyma and subcutaneous fat, or skin thickening. However, when it occurs in women middle-aged or older, associated malignancy should be considered. In our cases, we detected irregular hypoechoic malignant masses after the disappearance of inflammatory changes. Therefore, when non-puerperal women have inflammatory signs on their breast, follow-up imaging should be performed. In particular, in the case of persistent or growing palpability after the recovery of breast inflammation, percutaneous core biopsy and short-term follow-up with ultrasonography should be considered to exclude the associated malignancy.

Keywords: Breast; Mastitis; Breast neoplasms; Ultrasonography; Diagnosis

\section{Introduction}

Mastitis is an inflammatory condition of the breast. Its symptoms include sudden onset of pain and tenderness, swelling, erythema, warmth, and fever. Ultrasonography (US) is more useful than mammography for evaluating the lesions [1]. Mastitis causes edema with hyperechoic subcutaneous fat and skin thickening, which are visible on US. It is difficult to evaluate the deep portion of a large, edematous breast with a high-resolution transducer used for breast US due to limited penetration. Thus, the underlying breast cancer might be missed in these inflammatory conditions. Here, we report two cases of mastitis obscuring the pre-existing breast cancer.

\section{Case Reports}

Case 1

A 56-year-old woman visited our hospital for pain and a sensation of heat in the left breast. She had detected a palpable mass of the left breast 6 months earlier. However, she ignored the symptom and did not undergo any examinations. Three days before presentation to our hospital, she had a massage to loosen up the lump, and then the painful symptoms occurred.

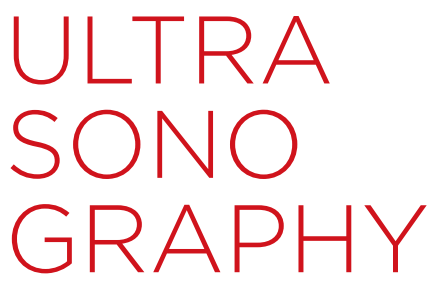

\section{CASE REPORT}

http://dx.doi.org/10.14366/usg.15024 pISSN: 2288-5919 e elSSN: 2288-5943 Ultrasonography 2016;35:159-163

Received: April 23, 2015

Revised: June 10, 2015

Accepted: June 11, 2015

Correspondence to: Jin Kyung An, MD, Department of Radiology, Eulji General Hospital, Eulji University School of Medicine, 68 Hangeulbiseok-ro, Nowon-gu, Seoul 01830, Korea

Tel. $+82-2-970-8290$

Fax. +82-2-970-8346

E-mail: jkan0831@eulji.ac.kr

This is an Open Access article distributed under the terms of the Creative Commons Attribution NonCommercial License (http://creativecommons.org/ licenses/by-nc/3.0/) which permits unrestricted noncommercial use, distribution, and reproduction in any medium, provided the original work is properly cited.

Copyright @ 2016 Korean Society of Ultrasound in Medicine (KSUM)

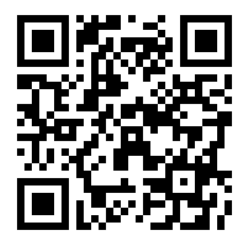

How to cite this article:

An JK, Woo JJ, Lee SA. Non-puerperal mastitis masking pre-existing breast malignancy: importance of follow-up imaging. Ultrasonography. 2016 Apr;35(2):159-163. 
US showed diffusely increased echogenicity in the subcutaneous fat and parenchyma with overlying skin thickening in the left upper and outer breast. Dilated lymphatics were seen in the subdermal layer. There were multiple tubular hypoechoic lesions and abscesslike lesions (Fig. 1A). The findings suggested an inflammatory condition such as granulomatous mastitis. Several enlarged lymph

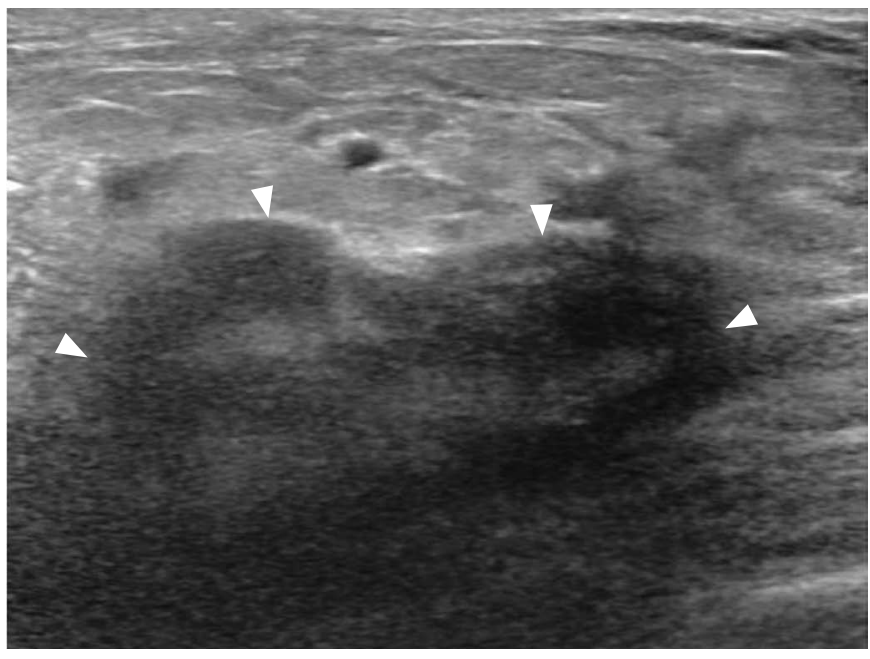

A

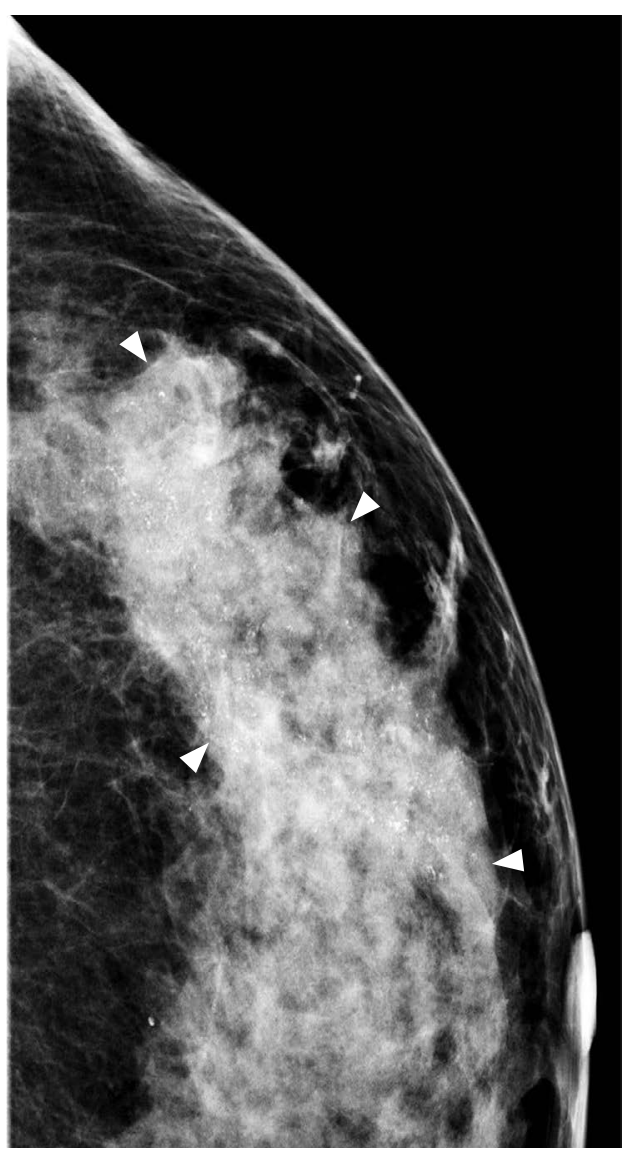

C

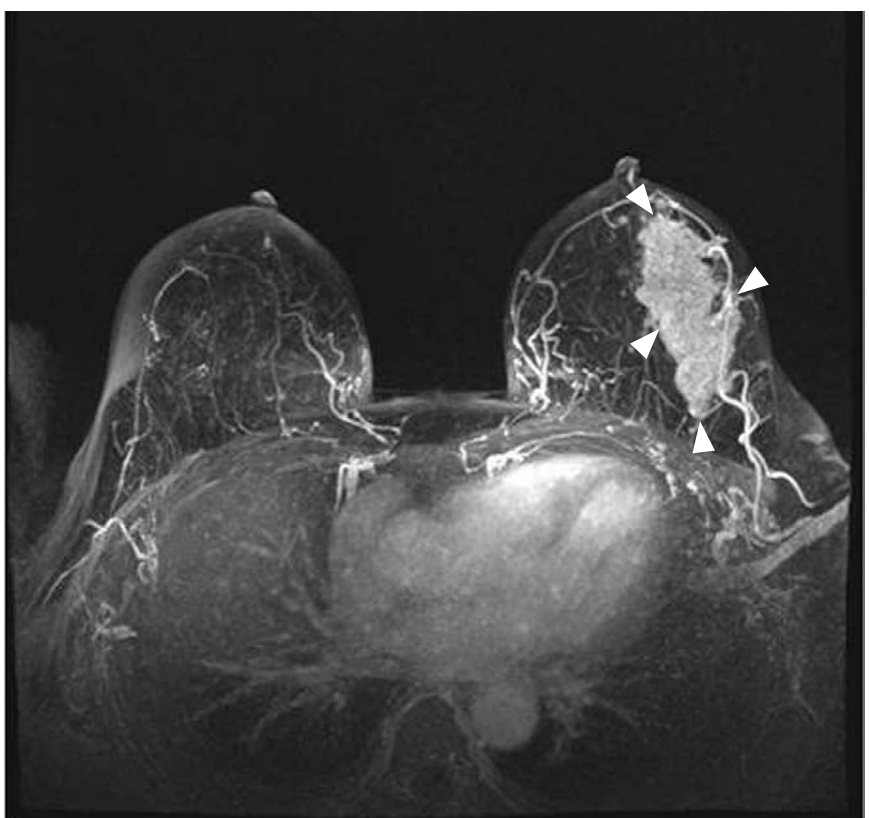

D

Fig. 1. A 56-year-old woman with the symptoms of left mastitis.

A. Sonogram shows a diffusely increased echo in subcutaneous fat and parenchyma with skin thickening. The engorged edematous breast and diffuse hyperechogenicity disturb the distinction of each structure of the breast. There are multiple tubular hypoechoic lesions in the left outer breast (arrowheads). B. Sonogram taken 3 weeks later shows the disappearance of the edema and skin thickening. However, diffusely distributed irregular hypoechoic lesions are revealed in the left outer breast with internal echogenic spots from microcalcifications (arrowheads). C. Mammogram shows an irregular hyperdense mass with a segmental distribution of microcalcifications in the left outer breast (arrowheads). D. Axial maximum intensity projection image shows an extensive enhancing lesion in the left outer breast (arrowheads). 
nodes were seen in the left axilla, suggesting reactive hyperplasia.

We first prescribed antibiotics and planned to biopsy the lesion in the case of lesion aggravation. The symptoms subsided several days later. At the 3-week follow-up, the symptoms completely disappeared. However, the palpable mass remained. Follow-up US showed improvement of edema and skin thickening. However, irregular hypoechoic lesions were diffusely distributed and microcalcifications were newly found in the left outer breast (Fig. 1B). Mammography showed irregular hyperdense masses with segmental microcalcifications in the left upper outer breast (Fig. 1C). US-guided core biopsy was performed. The lesion was diagnosed as ductal carcinoma in situ (DCIS) with focal suspicious invasion. Breast magnetic resonance imaging was conducted to evaluate the extent of the lesion, which showed extensive enhancement in the left outer breast (Fig. 1D). The patient underwent modified radical mastectomy. The pathology was $6.0 \mathrm{~cm} \times 5.0 \mathrm{~cm} \times 2.2 \mathrm{~cm}$ cribriform and comedo-type intraductal carcinoma with $1-\mathrm{mm}$ focal invasion and ductal extension of DCIS. There was no nodal metastasis. Immunohistochemical staining showed human epidermal growth factor receptor 2 (HER2)-enriched carcinoma that was HER2 3+, estrogen receptor-negative, and progesterone receptor-negative.

\section{Case 2}

A 63-year-old woman visited a breast clinic for pain, redness, and a sensation of heat in the left breast. The symptoms had suddenly appeared 3 days prior. She had no past medical history. US showed diffusely increased echogenicity of the left breast with mild skin thickening (Fig. 2A). Prominent duct ectasia was seen in the left subareola with some internal debris (Fig. 2B). There was no abscess formation. Several lymph nodes with mild cortical thickening were noted in the left axilla. She was prescribed antibiotics, and her symptoms subsided several days later.

Ten days later, after the pain subsided, she underwent mammography. The mammogram showed diffuse and trabecular thickening of the left breast and the left axillary lymphadenopathy (Fig. 2C). However, these mammographic findings were overlooked. Followup mammography after 6 months showed aggravation of trabecular thickening with the appearance of new masses in the left breast. US showed multiple irregular hypoechoic masses (Fig. 2D). Subareolar duct ectasia was still seen, but internal debris and adjacent inflammatory change disappeared (Fig. 2E). Cortical thickening of the left axillary lymph node was aggravated. She underwent USguided biopsy. The breast mass was confirmed as invasive ductal carcinoma. The left axillary lymphadenopathy was confirmed as a metastatic node.

\section{Discussion}

Mastitis is a breast inflammation that could be infectious or non-infectious. It could involve several distinct entities. Nonpuerperal mastitis is inflammation not related to pregnancy or lactation. Rupture of ectatic ducts or cysts can arise from chemical inflammation in the breast tissue, which can be superimposed by a secondary bacterial infection [2,3]. Mastitis can also be caused by direct trauma to the breast tissue caused by sports activities or a seat belt injury [4].

In case 1, as we initially thought that the massage could be the cause of inflammation for the 56-year-old woman, we decided to follow-up the symptoms with medication. Our differential diagnosis for the patient was granulomatous lobular mastitis because tubular hypoechogenicity and small abscess-like lesions were seen upon an edematous change. Pathologic features of granulomatous lobular mastitis are non-caseating granulomas and microabscesses confined to the breast lobule $[5,6]$. Young and parous women are frequently affected. However, it may occur in pre-pubertal and postmenopausal women [7]. The imaging findings are similar to breast malignancy. Biopsy is often needed to confirm the pathology. In our case, although the painful symptoms disappeared after medication, we should have performed follow-up US to exclude the possibility of malignancy. We missed the patient's history of " 6 -month-old palpable mass" in her breast. At the 3-week follow-up, after the resolution of combined edema and inflammatory changes, extensive hypoechoic lesions with microcalcifications were found.

In case 2, the 63-year-old woman had no palpable symptoms before the onset of inflammatory signs. On US, subareolar and central duct distension with internal debris were very prominent, leading us to consider non-puerperal mastitis. Diffusely increased echogenicity limited the evaluation of the deep portion of the breast. The patient also rapidly recovered from the symptoms after medication. We concluded it to be a case of simple mastitis. On mammography conducted 10 days later, we missed diffuse trabecular thickening and overlooked it as a part of heterogeneously dense parenchyma. The trabecular thickening in mammography was aggravated at the 6-month follow-up, which suggested that it was caused by a pre-existing malignancy instead of edema. In this case, the inflammation was a coincidental finding of breast malignancy.

Non-puerperal mastitis in association with non-inflammatory breast cancer is rare. Edey et al. [8] reported ductal breast carcinoma presenting with methicillin-resistant Staphylococcus aureus (MRSA) mastitis in a woman who had a wound infection by MRSA after a cesarean section. At that time, she had an episode of mastitis while breast feeding. Seventeen months later, after cessation of breast feeding, she presented with a 1-month history of mastitis in the 


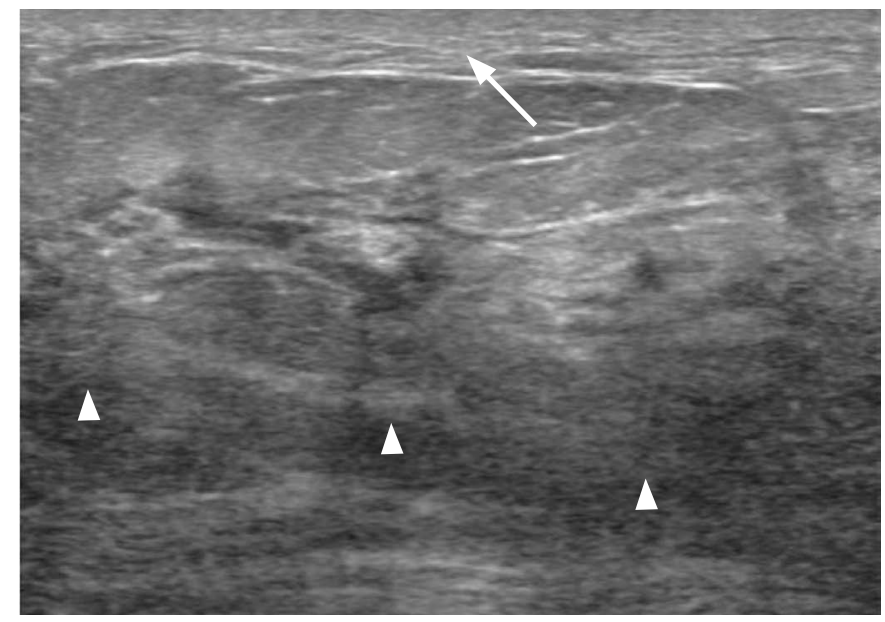

A

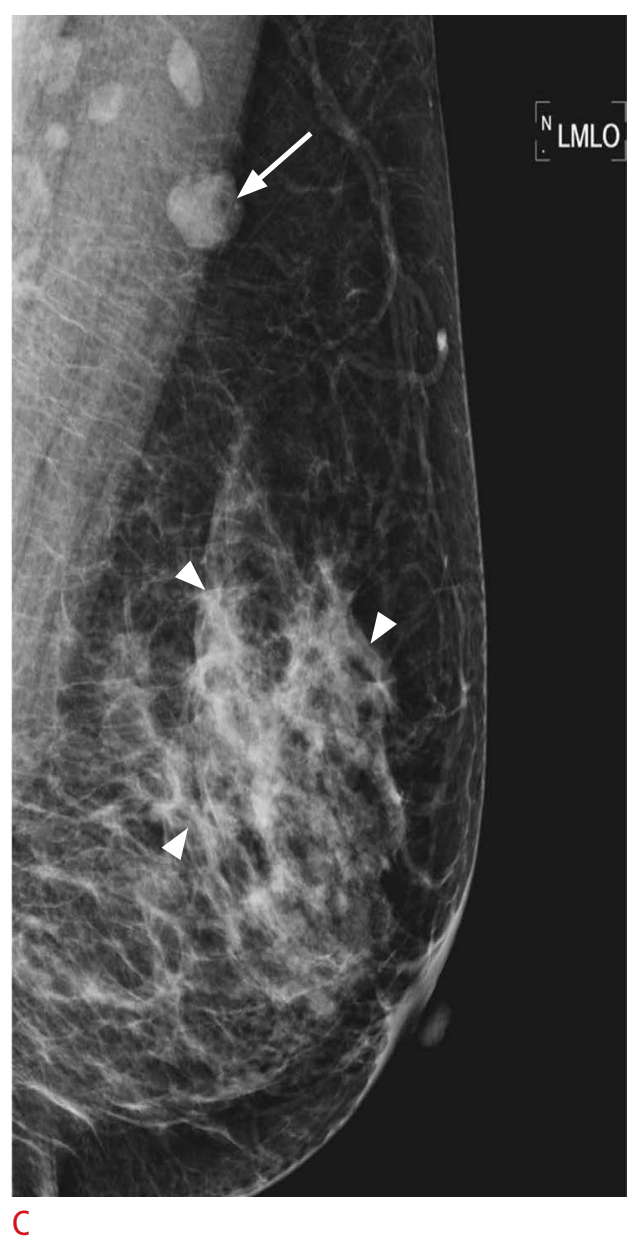

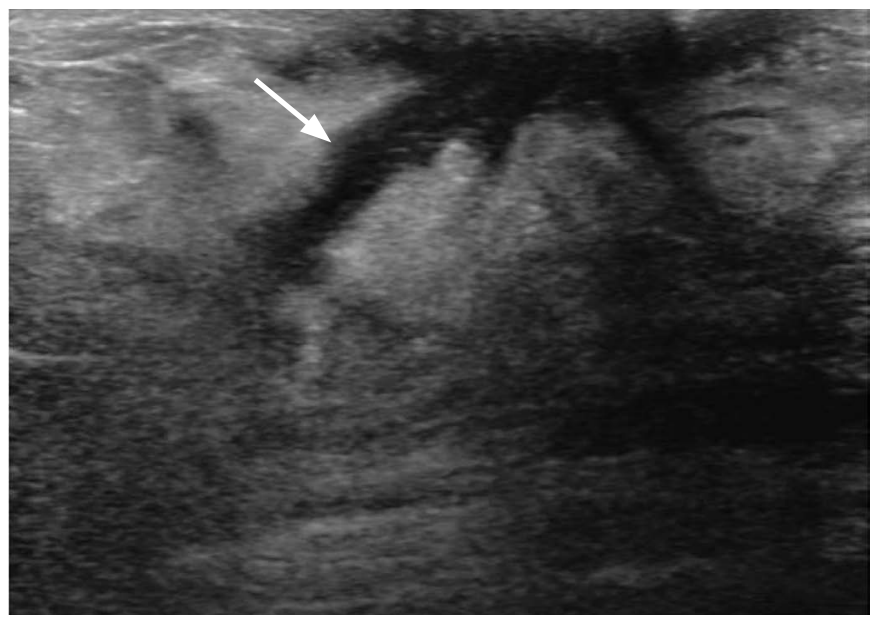

B

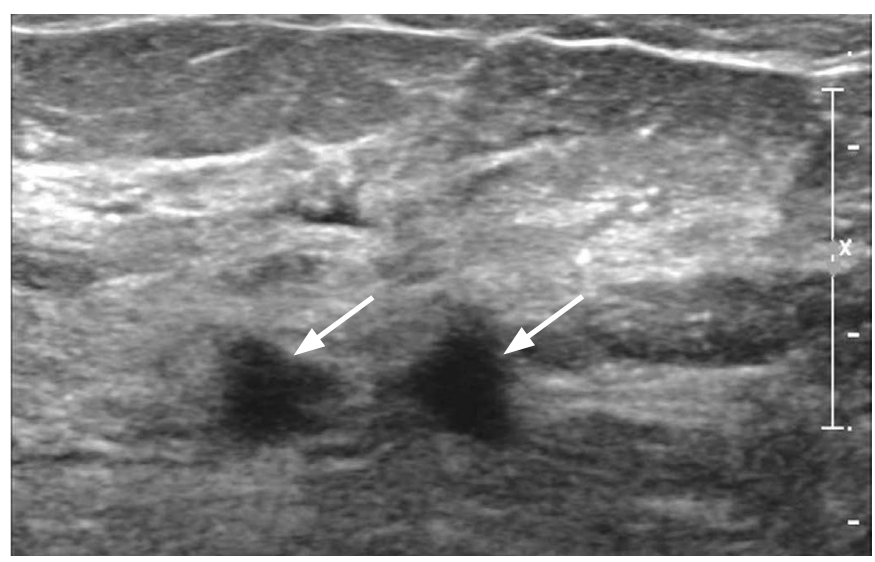

D

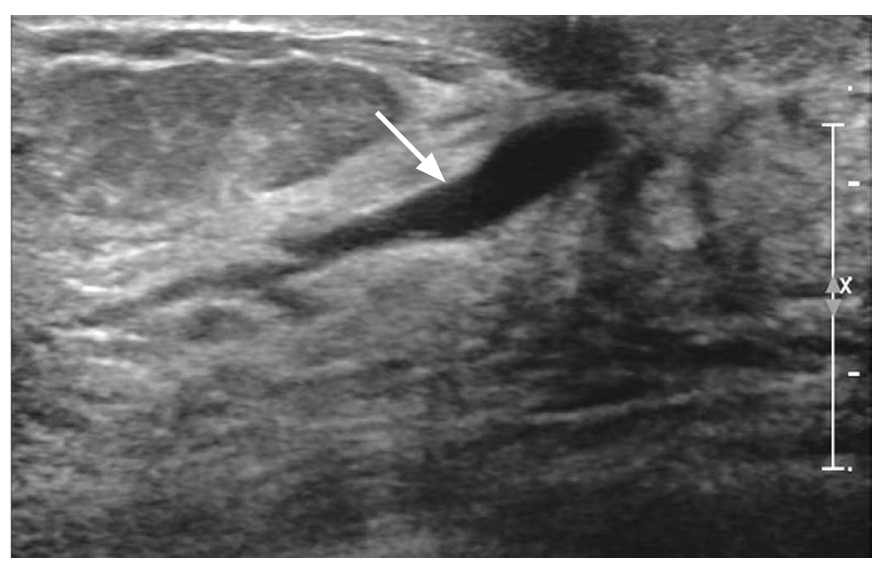

$\mathrm{E}$

Fig. 2. A 63-year-old woman with the symptoms of left mastitis.

A. Sonogram shows a diffusely increased echo of the left breast with mild skin thickening (arrow). Sonographic penetration is poor, and the distinction between retromammary fat and the parenchyma is not clear (arrowheads). B. Prominent duct distention is seen in the left subareola with some internal debris (arrow). C. Mammogram taken 10 days later shows diffuse and bizarre trabecular thickening of the left breast (arrowheads) and the left axillary lymphadenopathy (arrow). Skin thickening or haziness of the subcutaneous fat is not present. D. Multiple irregular hypoechoic masses are seen in the deep layer of the left breast (arrows). Previously increased echogenicity of the fat and skin thickening disappears. The distinction of subcutaneous fat and parenchyma is very clear. E. Subareolar duct distension is still seen, but internal debris and adjacent inflammatory changes are gone (arrow). 
same breast. MRSA was cultured from a skin swab. An imaging study revealed suspicious calcifications. She underwent surgery after being diagnosed with multifocal ductal carcinoma with high-grade DCIS. The authors explained that the necrotic ductal tissue from high-grade DCIS might have acted as a nidus for a chronic infection of MRSA.

Mastitis must be distinguished from inflammatory breast cancer manifested as severe edema, erythema, and heating sensations involving more than one-third of the skin of the breast associated with invasive carcinoma [9-11]. The diagnosis of inflammatory breast cancer is made on the basis of the characteristic skin features and pathology confirmation of the breast or skin showing invasive ductal carcinoma. Benign mastitis and inflammatory breast cancer have similar symptomatic features. However, mastitis may respond to antibiotics, whereas an inflammatory breast does not.

In conclusion, US is useful for the monitoring of skin thickening, breast parenchymal changes, and abscess formation. Mammography should be performed after the pain subsides because it is superior to other modalities in evaluating calcifications. When non-puerperal women have mastitis, follow-up imaging should be performed. In particular, in women who have persistent or growing palpability after the recovery of breast inflammation, percutaneous core biopsy and short-term follow-up with US should be performed to exclude the associated malignancy.

ORCID: Jin Kyung An: http://orcid.org/0000-0003-1086-426X; Jeong Joo Woo: http://orcid.org/0000-0002-4669-6176; Seung A Lee: http://orcid.org/0000-00032435-1254

\section{Conflict of Interest}

No potential conflict of interest relevant to this article was reported.

\section{References}

1. Boisserie-Lacroix M, Lafitte JJ, Sirben C, Latrabe V, Grelet P, Zeinoun $R$, et al. Inflammatory and infectious lesions of the breast: contribution of ultrasonography. J Chir (Paris) 1993;130:408-415.

2. Maier WP, Berger A, Derrick BM. Periareolar abscess in the nonlactating breast. Am J Surg 1982;144:359-361.

3. Berna-Serna JD, Berna-Mestre JD. Follicular occlusion due to hyperkeratosis: a new hypothesis on the pathogenesis of mammillary fistula. Med Hypotheses 2010;75:553-554.

4. Greydanus DE, Omar H, Pratt HD. The adolescent female athlete: current concepts and conundrums. Pediatr Clin North Am 2010;57:697-718.

5. Page DL, Anderson TJ. Diagnostic histopathology of the breast. Edinburgh: Churchill Livingstone, 1987;64-65.

6. Symmers WS. Systemic pathology. 3rd ed. Edinburgh: Churchill Livingstone, 2000;192-195.

7. Jorgensen MB, Nielsen DM. Diagnosis and treatment of granulomatous mastitis. Am J Med 1992;93:97-101.

8. Edey AJ, Bentley PG, Garrett JP, Liebmann RD. Ductal breast carcinoma presenting with methicillin-resistant Staphylococcus aureus mastitis. Breast I 2005;11:491-492.

9. Rosen PP. Invasive mammary carcinoma. In: Harris JR, Lippman ME, Morrow M, Hellman S, eds. Diseases of the breast. Philadelphia: Lippincott-Raven, 1996;393-444.

10. Dershaw DD, Moore MP, Liberman L, Deutch BM. Inflammatory breast carcinoma: mammographic findings. Radiology 1994;190: 831-834.

11. Tardivon AA, Viala J, Corvellec Rudelli A, Guinebretiere JM, Vanel D. Mammographic patterns of inflammatory breast carcinoma: a retrospective study of 92 cases. Eur J Radiol 1997;24:124-130. 